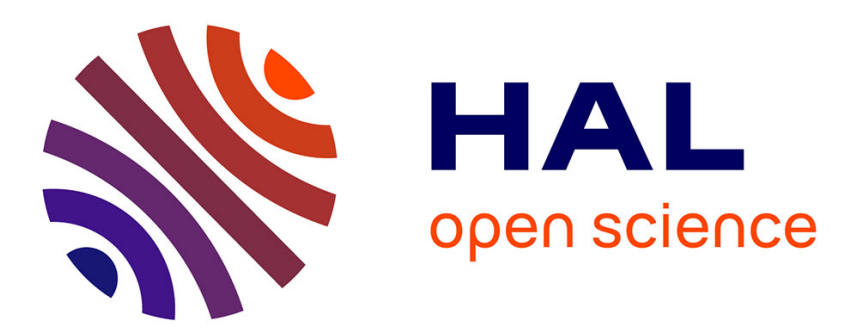

\title{
Experimental evidence of enhanced electro-optic control on a lithium niobate photonic crystal superprism
}

\author{
J. Amet, G. Ulliac, F.I. Baida, M.-P. Bernal
}

\section{To cite this version:}

J. Amet, G. Ulliac, F.I. Baida, M.-P. Bernal. Experimental evidence of enhanced electro-optic control on a lithium niobate photonic crystal superprism. Applied Physics Letters, 2010, 96, pp.103111. 10.1063/1.3359421 . hal-00463178

\section{HAL Id: hal-00463178 https://hal.science/hal-00463178}

Submitted on 12 Apr 2021

HAL is a multi-disciplinary open access archive for the deposit and dissemination of scientific research documents, whether they are published or not. The documents may come from teaching and research institutions in France or abroad, or from public or private research centers.
L'archive ouverte pluridisciplinaire HAL, est destinée au dépôt et à la diffusion de documents scientifiques de niveau recherche, publiés ou non, émanant des établissements d'enseignement et de recherche français ou étrangers, des laboratoires publics ou privés. 


\title{
Experimental evidence of enhanced electro-optic control on a lithium niobate photonic crystal superprism
}

\author{
J. Amet, G. Ulliac, F. I. Baida, and M.-P. Bernal ${ }^{a)}$ \\ Département d'Optique, Institut FEMTO-ST, CNRS UMR6174, Université de Franche-Comté, \\ 16 Route de Gray, 25030 Besançon Cedex, France
}

(Received 9 December 2009; accepted 17 February 2010; published online 10 March 2010)

\begin{abstract}
We present experimental evidence of an electro-optic superprism photonic crystal fabricated on a lithium niobate substrate, in agreement with simulation results. In this work, rather than changing the incident wavelength or angle to achieve large light deflections, a direct modification of the band structure by the electro-optic effect causes changes in the propagation direction of the incident beam. Additionally, the chosen geometry allows significant ultrarefraction as the incident wavelength is varied giving rise to a double purpose active-passive photonic crystal light deflector that could present potential interest for optical communications. (c) 2010 American Institute of Physics. [doi:10.1063/1.3359421]
\end{abstract}

Since the introduction of the photonic crystal (PC) concept in 1987, photonic crystals have become widely studied by the optics community. PCs capabilities allow light control on propagation distances of only several wavelengths and many applications like perfect mirrors, ${ }^{1,2}$ photonic crystal waveguides, ${ }^{3,4}$ and microcavities ${ }^{5,6}$ to confine light, to guide light, or to inhibit or amplify spontaneous emission properties have emerged. Among of the PC applications, the superprism (SP) effect offers a largely enhanced angular dispersion in comparison to bulk materials. This effect does not appear inside the photonic band gap, but in certain propagation bands possessing starlike isofrequency contours. The SP effect can be explained by examination of the highly anisotropic dispersion surface found in photonic crystals.

Tunable SP PCs structures combine high angular dispersion with optically active media to achieve spatial beam deflection. While active tuning on the band gap position has been theoretically and experimentally demonstrated by many authors, tunable SPs have been only proposed numerically. ${ }^{7-10}$

In this work, we show experimental evidence, thanks to scanning near field imaging, of an electro-optical photonic crystal SP. The applied voltage needed is greatly reduced with respect to bulk lithium niobate material thanks to a geometry that has been looked for allowing slow light propagation as well as ultrarefraction. The geometry has been found with a systematic planar wave expansion (PWE) numerical study.

In a first place, a suitable geometry has been searched for. Therefore, we are going to calculate a design metric that will pin point a set of parameters for which ultrarefraction and slow light are both present. Thus, our design geometry paves the way to a device that can be used in three operating modes: Passive (that is, classical wavelength ultrarefraction), active (slow light enhanced electro-optical ultrarefraction) and the combination of both. We have chosen a triangular lattice of air holes $(n=1)$ in a X-cut lithium niobate annealed proton exchange (APE) waveguide $\left(\mathrm{n}_{\mathrm{LN}}=2.143\right)$. Two parameters are studied, the incident angle $\Theta_{\text {in }}$, and the normalized frequency $\Omega=a / \lambda$. Holes sizes have been chosen so that

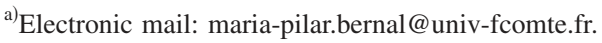

$\mathrm{r} / \mathrm{a}=0.2$ ( $\mathrm{r}=$ hole radius, $\mathrm{a}=$ lattice periodicity). The lattice is oriented in the $\Gamma \mathrm{K}$ direction as shown in Fig. 1. Given that the APE waveguides only allow transverse electric polarization (electric field perpendicular to the hole axis), the numerical simulations are performed only with this polarization state. The incident beam is a plane wave propagating perpendicular to the air hole axis. As far as PWE simulations are concerned, the photonic crystal is considered infinite in both directions of the propagating plane and the holes have infinite depth.

For each set of parameters, we compute group velocity propagation direction $\Theta_{\mathrm{vg}}$ and the local field factor for two refractive indexes $\mathrm{n}_{1}=\mathrm{n}_{\mathrm{LN}}=2.143$, and $\mathrm{n}_{2}=2.16$ (band edge), which corresponds to a modification of the APE lithium niobate substrate of $\Delta \mathrm{n}=0.017$. This modification can be achieved by different physical phenomena. In the present work, the modification is obtained thanks to the Pockels effect of lithium niobate.

The computation results can be analyzed in the form of two-dimensional design maps. As in Ref. 11, we have computed a design metric defined as,

$$
\log \left(\frac{f_{1}+f_{2}}{2}\left|\Theta_{\mathrm{v}_{g_{1}}}-\Theta_{\mathrm{v}_{g_{2}}}\right|\right) \text {, }
$$

where $\mathrm{f}_{1}$ and $\mathrm{f}_{2}$ are the local field factors and $\Theta_{\mathrm{vg} 1}$ and $\Theta_{\mathrm{vg} 2}$ the group velocity directions for the refractive indexes $n_{1}$ and

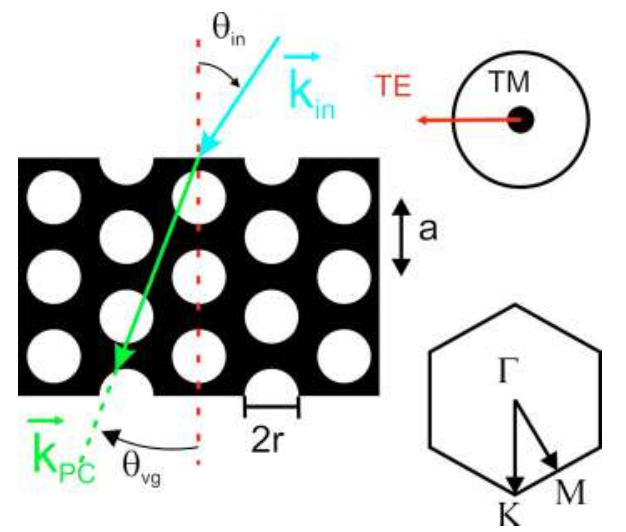

FIG. 1. (Color online) Schematic of the Brillouin zone and the PC lattice in the real space. 


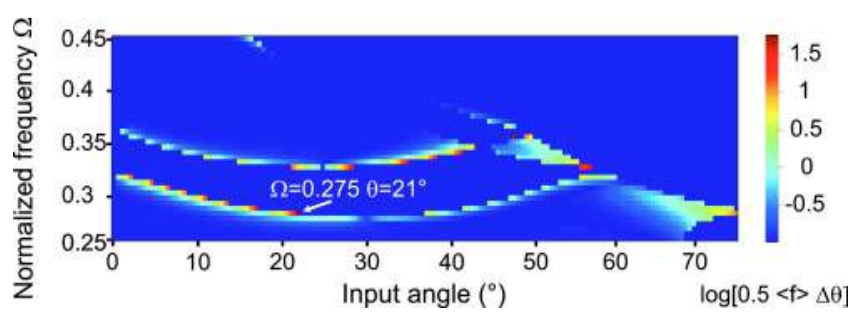

FIG. 2. (Color online) Design metric for slow light and ultrarefraction with $\mathrm{r} / \mathrm{a}=0.2$ and $\Gamma \mathrm{K}$ lattice termination.

$\mathrm{n}_{2}$, respectively. This equation quantifies the idea that we are searching design configurations where the group velocity stays low (high $\mathrm{f}$ values) and simultaneously, the electrooptic beam steering is large.

Results are shown in Fig. 2. The colormap shows both excellent (in red) and fair (green and yellow) design solutions. It can be observed that several regions (red) present strong slow light electro-optic ultrarefraction. We pay particular attention to the configuration corresponding to $\Omega$ $=0.275$, and $\Theta_{\text {in }}=21^{\circ}$. Since we are interested in the telecom wavelength range centered on $\lambda=1550 \mathrm{~nm}$, the periodicity must be set to $\mathrm{a}=430 \mathrm{~nm}$.

This particular configuration works also in a passive mode, that is, the beam steering is modified by the incident wavelength. PWE simulations of the passive device have been reported in Ref. 12, and predict a beam steering of $4.3 \% \mathrm{~nm}$ for a wavelength range between 1547 and $1562 \mathrm{~nm}$. The experimental results show a more modest steering of $1.5 \% \mathrm{~nm}$ for a wavelength range from 1556 and $1572 \mathrm{~nm}$. The difference between the simulations and the experiments has been attributed to the finite size of the photonic crystal, which is not taken into account by the two-dimensional PWE simulations. This observation has also been reported by other authors. ${ }^{8,13}$

Let us now study the electro-optic tunability of the beam angle propagating in the photonic crystal. In order to use low external voltages, a slow light geometry is also needed. Figure 3 displays the PWE simulation results which show the dependence of the local field factor, $\mathrm{f}$, and group velocity direction, $\Theta_{\mathrm{vg}}$, as a function of the substrate index of refraction. The wavelength chosen is $1565 \mathrm{~nm}$. The figure shows that, in the case of a monochromatic wave, if the index of refraction varies from $n_{1}$ to $n_{2}$, the group velocity direction changes from $2^{\circ}$ to $-44^{\circ}$ and the corresponding local field

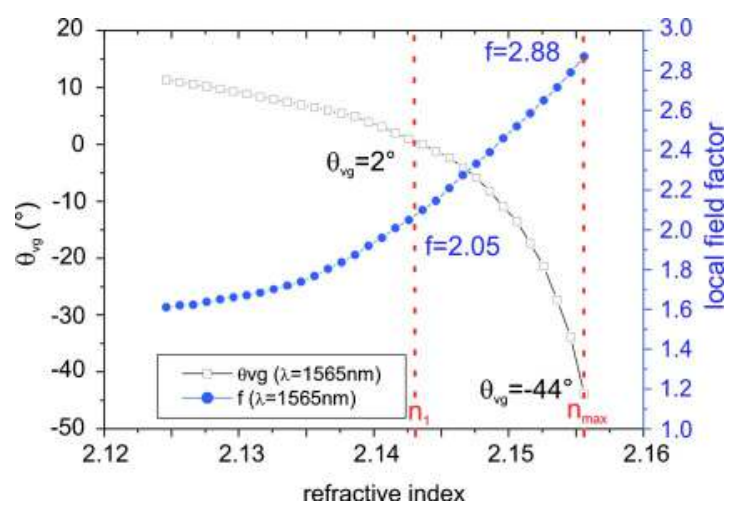

FIG. 3. (Color online) Angle of the group velocity vector and local field factor, $\mathrm{f}$, within a lithium niobate triangular lattice photonic crystal as a function of refractive index. (a)

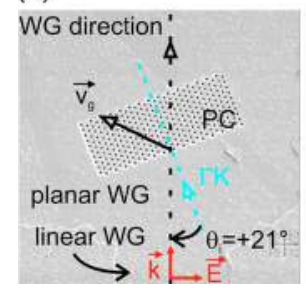

(b)

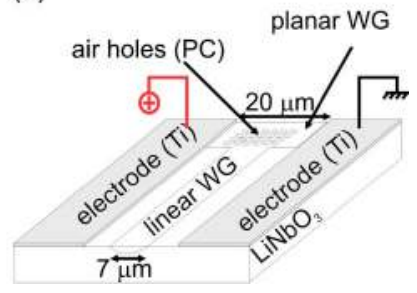

FIG. 4. (Color online) Schematics of the designed device and its corresponding SEM image.

factor values are $\mathrm{f}_{1}=2.05$ and $\mathrm{f}_{2}=2.88$, respectively, giving a mean value of the local field factor of 2.45 . If we compare with the Pockels effect for bulk lithium niobate, this particular photonic crystal geometry allows, for a given external applied voltage, an enhancement of the index of refraction variation of $14.7\left(\mathrm{f}^{3}\right)$. Thus, only $5.8 \mathrm{~V} / \mu \mathrm{m}$ are necessary to obtain an index variation of $\Delta n=0.013$. Since, according to the results shown in Fig. 3 the local field factor is not constant with respect to the index of refraction but it increases as it approaches the band edge $\left(n=n_{2}\right)$, we should find a better SP performance as the band edge is approached.

The next step is to validate the simulated results experimentally. The photonic crystal, a triangular lattice of 10 $\times 25$ holes with $\mathrm{a}=430 \mathrm{~nm}$ and $\mathrm{r}=86 \mathrm{~nm}$, was fabricated by focused ion beam milling as detailed in Ref. 14. Other alternative fabrication methods based on domain inversion have been reported elsewhere. ${ }^{15-17}$ The light confinement in the vertical direction is guaranteed by etching the lattice on an annealed proton exchange lithium niobate waveguide. The incident beam is tilted $21^{\circ}$ with respect to the $\Gamma \mathrm{K}$ direction. Titanium electrodes of $100 \mathrm{~nm}$ thickness and a separation of $20 \mu \mathrm{m}$ are deposited in both sides along the photonic crystal as show schematically in Fig. 4. Light exits the APE waveguide $100 \mu \mathrm{m}$ away from the photonic crystal.

In order to visualize the light deviation inside the photonic crystal a scanning near field optical microscope (SNOM) in transmission mode is used. A dielectric fibered tip collecting the evanescent field scans the SP surface in the direction perpendicular to the APE waveguide. The injected wavelength is $1565 \mathrm{~nm}$. The optical near field intensities normalized by the maximum of the transmitted signal outside the photonic band gap are displayed in Fig. 5 for two external voltages, 0 and $-100 \mathrm{~V}$. The cross-sections of the intensities taken at a distance of $1.5 \mu \mathrm{m}$ with respect to the photonic crystal entrance plane are also represented. The incident beam is represented with a white arrow and the PC boundaries are drawn with a discontinuous white line. At $0 \mathrm{~V}$ the beam deviation is of $-3^{\circ} \pm 1.8^{\circ}$ and at $-100 \mathrm{~V}$ of $17^{\circ} \pm 7.1^{\circ}$, both angles measured with respect to the $\Gamma \mathrm{K}$ direction. Please note that the cross-section angles in Figs. 5(c) and 5(d) are mean values and are displayed with respect to the incident beam axe (white arrow). From this values and, taken into account that we know that at $0 \mathrm{~V}$ the index of refraction is the one corresponding to the APE waveguide, that is 2.143 , we can, by using the PWE simulations, obtain an estimation of the electro-optic enhancement on the photonic crystal.

First, we can observe that at $0 \mathrm{~V}$, the experimental and theoretical beam deviation values present excellent agreement $\left(2^{\circ}\right.$ according to PWE and, $-3^{\circ} \pm 1.8^{\circ}$ with measured by SNOM). The application of $-100 \mathrm{~V}$ has steered the beam 
(a)

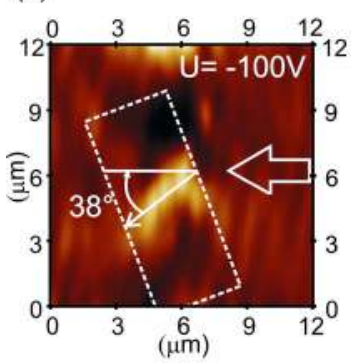

(c)

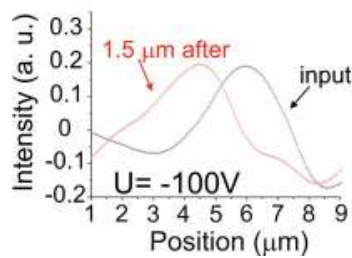

(b)

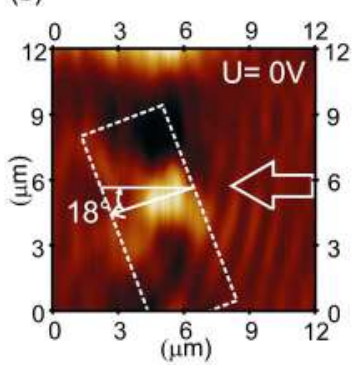

(d)

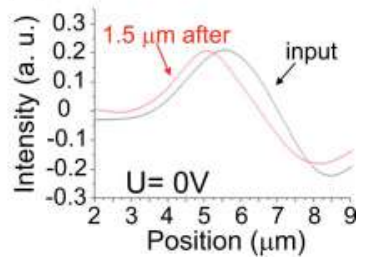

FIG. 5. (Color online) Scanning near field optical images of light propagating inside the photonic crystals at $-100 \mathrm{~V}$ (a) and $0 \mathrm{~V}$ (b) and the corresponding cross-sections $[(\mathrm{c})$ and (d)] taken at $1.5 \mu \mathrm{m}$ away from the photonic crystal entrance, and perpendicular to the $\Gamma \mathrm{K}$ direction.

to the angular position $17^{\circ} \pm 7.1^{\circ}$ due to an index variation of 0.01 (changing therefore to an index of refraction value of 2.153). If we would apply Pockels equation for bulk lithium niobate, such index variation would have needed an external voltage $1320 \mathrm{~V}$ instead of $100 \mathrm{~V}$. The enhancement factor is therefore of the order of 13.2 which corresponds to a measured local field factor of $\mathrm{f}_{\mathrm{EXP}}=2.35$, which shows good agreement with the PWE numerical simulations ( $f_{\mathrm{PWE}}$ $=2.45$ ). This enhancement is due to the fact that light is slowly propagating inside the photonic crystal which has an effect as if the electro-optic coefficient was larger. ${ }^{18}$

In conclusion, we have experimentally demonstrated an electro-optical tunable photonic crystal SP in lithium niobate. A geometry allowing ultrarefraction and slow-light propagation has been found thanks to a systematic PWE numerical study. Due to the slow photons, the external voltage needed to have a mean beam steering of $20^{\circ}$ is $100 \mathrm{~V}$, a value 13 times smaller than the estimated by the electro-optic effect in bulk lithium niobate. This tunable SP in lithium niobate could find applications in the optical telecommunications and integrated optics.

The authors are grateful to EU for its financial support under Grant No. STREP 033297 3D-DEMO, to S. Diziain for contributing to the SNOM measurements, and to G.W. Burr (IBM, Almaden Research Center) for helpful discussions.

${ }^{1}$ J. D. Joannopoulos, P. Villeneuve, and S. Fan, Nature (London) 386, 143 (1997).

${ }^{2}$ J. D. Joannopoulos, J. Meade, and J. N. Winn, Photonic Crystals: Molding the Flow of Light (Princeton University Press, New Jersey, 1995).

${ }^{3}$ S. Assefa, S. J. McNab, and Y. A. Vlasov, Opt. Lett. 31, 745 (2006).

${ }^{4}$ M.-P. Bernal, N. Courjal, J. Amet, M. Roussey, and C. Hou, Opt. Commun. 265, 180 (2006).

${ }^{5}$ O. Painter, R. K. Lee, A. Yariv, A. Scherer, J. O'brien, P. D. Dapkus, and I. Kim, Science 284, 1819 (1999).

${ }^{6}$ T. Asano, B.-S. Song, and S. Noda, Opt. Express 14, 1996 (2006).

${ }^{7}$ G. Alagappan, X. W. Sun, and P. Shum, Opt. Lett. 31, 1109 (2006).

${ }^{8}$ D. Scrymgeour, N. Malkova, S. Kim, and V. Gopalan, Appl. Phys. Lett. 82, 3176 (2003).

${ }^{9}$ N. C. Panoiu, M. Bahl, and R. M. Osgood, Jr., Opt. Lett. 28, 2503 (2003).

${ }^{10}$ T.-H. Pei and Y.-T. Huang, Jpn. J. Appl. Phys., Part 2 46, L593 (2007).

${ }^{11}$ J. Amet, F. I. Baida, G. W. Burr, and M.-P. Bernal, Photonics Nanostruct. Fundam. Appl. 6, 47 (2008).

${ }^{12}$ S. Diziain, J. Amet, F. I. Baida, and M. P. Bernal, Appl. Phys. Lett. 93, 261103 (2008).

${ }^{13}$ D. Bernier, E. Cassan, A. Lupu, G. Maire, D. Marris-Morini, L. Vivien, and S. Laval, Opt. Commun. 274, 241 (2007).

${ }^{14}$ F. Lacour, N. Courjal, M.-P. Bernal, A. Sabac, C. Bainier, and M. Spajer, Opt. Mater. 27, 1421 (2005).

${ }^{15}$ A. C. Busacca, C. L. Sones, V. Apostolopoulos, R. W. Eason, and S. Mailis, Appl. Phys. Lett. 81, 4946 (2002).

${ }^{16}$ S. Grilli, P. Ferraro, P. De Natale, B. Tiribilli, and M. Vassalli, Appl. Phys. Lett. 87, 233106 (2005).

${ }^{17}$ P. Ferraro and S. Grilli, Appl. Phys. Lett. 89, 133111 (2006).

${ }^{18}$ M. Roussey, F. I. Baida, and M.-P. Bernal, J. Opt. Soc. Am. B 24, 1416 (2007). 